intervention and the labelling of a large number of normal infants with a condition they do not have.

Before embarking on such a course the baby should at the very least be observed, handled, and fed by experienced staff in a calm atmosphere, perhaps in a day care unit. This often resolves the problem and relieves the parents of much unnecessary anxiety. If this fails to resolve symptoms I concede that a trial with soy might be worthwhile in those few babies whose symptoms remain, but there is no convincing objective evidence that this is beneficial. Perhaps someone should try to find out.

\section{Screening for cystic fibrosis}

Sir,

In his commentary on our recent review paper $^{1}$ in which we advocate a programme of neonatal screening for cystic fibrosis (CF), Professor Peter Phelan rightly points out that data in the published reports are inconclusive. This is partly because of the difficulty in obtaining data which compare like populations. Professor Phelan himself compares the survival figures for 30 years ago (fewer than $10 \%$ alive at 5 years) when definitive diagnosis was often made at necropsy only, with major contemporary US and Australian statistics showing $80 \%$ survival to late adolescence. The Gibson-Cooke standardised sweat test was not available before 1959, and its widespread application has resulted in identification of milder cases and better understanding of the considerable variations in the natural history of CF which are independent of particular treatment regimens.

Our support for a neonatal screening programme is based as much on the value of a pre-symptomatic diagnosis to the individual parents and child as on the scanty useful incidence and mortality data in the reports. ${ }^{2}$ The fact that the majority of newly diagnosed children with CF are born into families without a previously affected child is of no help to those parents whose eldest CF child is diagnosed only after the birth of the second. We are less sanguine about the long term outlook for CF patients than Professor Phelan and conscious of the physical and emotional cost at which the improving prognosis is achieved. New approaches to treatment are needed, perhaps with particular attention to early nutritional support. A recent paper from another Australian centre indicates how, even in patients with advanced CF, substantial benefit can be achieved by an aggressive nutritional policy. ${ }^{3}$ We have not, contrary to the fear expressed by Professor Phelan, encountered parental rejection on the grounds of the diagnosis of CF. Our experience is that parents told (we hope sensitively, optimistically, but realistically) that their new born infant has CF nearly always form a very close bond with their baby: nor is rejection an observed phenomenon in the $15 \%$ or so who present with meconium ileus and are effectively self selected for neonatal screening.

We agree that measures of the progress of lung disease in early life are insensitive, and feel that Professor Phelan can not really be sure that his 39 patients with a family history, who were therefore detected early, were as- certained 'before the development of lung disease'. We also agree that the benefits of early diagnosis in a long term prospective study of survival may be small, but this is not a valid argument against such a study since only an unselected series can unequivocally show differences between centres and treatment regimens-the methods of selection for treatment at a particular centre being otherwise questionable.

The existence of a screening programme implies the existence of a regional centre for diagnosis with which a treatment centre is likely to be associated. Although Professor Phelan claims that $95 \%$ of all patients in Victoria are treated at his own (justly renowned) centre, simple population statistics suggest that this may not be so. In our own centre, which treats all patients from South Glamorgan (population approximately 400000 ) and an equal number from other health authorities, about 55 are resident in the area. This gives a prevalence of 1:7500. The population of Victoria is about 4000000 and the clinic in Melbourne has 336 patients, ie a prevalence of $1: 12000$ in a comparable ethnic group. Either the Cardiff survival figures are much better than those of Victoria (which we doubt) or about $30 \%$ of the Australian patients are being cared for, or dying undiagnosed, without reference to the regional centre. Perhaps there is therefore a particularly strong case for neonatal screening in Victoria, the results of which might well surprise us all.

\section{References}

1 Phelan P D. Commentary. Arch Dis Child 1982; 57: 779-80.

2 Dodge J A, Ryley H C. Screening for cystic fibrosis. Arch Dis Child 1982; 57: 774-80.

3 Shepherd R, Cooksley W G E, Cooke W D D. Improved growth and clinical, nutritional, and respiratory changes in response to nutritional therapy in cystic fibrosis. J Pediatr 1980; 97 : 351-7.

J A DODGE AND H C RYLEY

Departments of Child Health and Medical Microbiology, University of Wales, Heath Park, Cardiff CF4 $4 X N$

\section{Professor Phelan comments:}

In their letter Dodge and Ryley raise a number of important issues that warrant further comment. I am pleased that they agree that great difficulties are likely to be experienced in showing an improved outlook as a result of newborn screening for cystic fibrosis and they are almost certainly correct in predicting that demonstrable long term benefits of early diagnosis will, at the best, be small.

They suggest that parents appreciate early presymptomatic diagnosis. However, this remains to be proved by a properly conducted study. It is not reasonable to compare ill babies with meconium ileus with those diagnosed pre-symptomatically. It seems crucial to show, before newborn screening is widely introduced, that pre-symptomatic diagnosis is beneficial to parents and child and that it does not disturb developing family 
relationships. It is to be hoped that such a study will be conducted in association with one of the pilot screening programmes under way in a number of countries so that assessment of value and harm can be based on fact rather than on opinion.

While it may be a tragedy that some families discover that an older child has CF only after the birth of a second affected child, this must be seen in perspective. As stated in my commentary, only $7 \%$ of patients are so diagnosed and 1 in 3 families who find they have 2 CF children in this way, elect to have further children, knowing fully the risks. Emotional concern for an individual family should not bias our ability to look objectively at the total problem.

Dodge and Ryley state that the Gibson-Cooke method of standardised sweat test was not available before 1959.1 However, Anderson and Freeman ${ }^{2}$ introduced collection of sweat for analysis of sodium and chloride, using a method not dissimilar to that subsequently described by Gibson and Cooke, at the Royal Children's Hospital in Melbourne in February 1956. Therefore, our patients have been diagnosed in this way for the last 27 years.

Drs Dodge and Ryley give some interesting figures on the prevalence of cystic fibrosis in South Glamorgan and suggest this is considerably higher than that of recognised cases in Victoria. Regretably they do not give the ages of the patients to whom they refer. If the 60 patients aged over 18 years resident in Victoria and attending an adult clinic are included, the prevalence in the two communities is not notably different. However, prevalence is not the appropriate method of measuring the frequency of a congenital disorder that is life limiting. The proper measurement is incidence related to live births and in Victoria since 1955 this has been $1: 2556^{3}$ It did not change appreciably between 1955 and 1978, suggesting that there was not a marked increase in identification of milder cases. Data reported elsewhere indicate that it is highly unlikely that a number of cases are being missed. ${ }^{4}$ Further, a study of newborn screening, using serum IRT, is currently under way in New South Wales, the adjacent state to Victoria in Australia. Incidence based on the first 75000 tests, and allowing for one false negative, was $1: 2083$ live births $(95 \%$ confidence limits $1: 1562$ to $1: 3125) .{ }^{5}$ Thus the incidence is not significantly different from that in the much longer study in Victoria. There is no support for the suggestion by Ryley and Dodge that $30 \%$ of patients in Victoria are being cared for or dying undiagnosed without reference to the regional centre.

The value and possible harm from newborn screening for CF remain unproven. Until well supported data are available screening programmes should continue to be seen as research endeavours and it would seem unwise to introduce them nationally.

\section{References}

1 Gibson L E, Cooke R E. A test for concentration of electrolytes in sweat in cystic fibrosis of the pancreas utilizing pilocarpine by iontophoresis. Pediatrics 1959; 23: 545-9.

2 Anderson C M, Freeman M. A simple method of sweat collection with analysis of electrolytes in patients with fibrocystic disease of the pancreas, and their families. Med J Aust 1958; i : 419-22.
3 Allan J L, Robbie M, Phelan P D, Danks D M. The incidence and presentation of cystic fibrosis in Victoria 1955-1978. Aust Paediatr J 1980; 16: 270-3.

4 Danks D M, Allan J L, Phelan P D, Chapman C. Mutations at more than one locus may be involved in cystic fibrosis-evidence based on first cousin data and direct counting of cases. Am J Hum Genet 1983; in press.

5 Wilcken B, Brown A R D, Urwin R, Brown D A. Cystic fibrosis screening by blood spot trypsin assay: results in 75,000 newborn infants. $J$ Pediatr 1983 ; in press.

\section{Neonatal systemic candidiasis}

Sir,

McDougall et al. ${ }^{1}$ reported the failure of intravenous miconazole in two preterm infants with systemic candidiasis, who subsequently responded to the combined therapy of amphotericin B and flucytocine. These two antifungal agents were not used at first because of reports of nephrotoxicity, hypokalaemia, hypotension, and thrombocytopenia secondary to amphotericin B, and thrombocytopenia, leucopenia and rashes and the development of resistance to flucytocine. ${ }^{2-4}$

During the last 3 years 7 infants have developed systemic fungal infection in this unit and all have survived. In all cases except 1 , the infecting organism was Candida albicans, the exception being Torulopsis glabrata. At the time of infection all infants were receiving parenteral nutrition; 6 were preterm, gestational age $27 \cdot 4 \pm 1 \cdot 8$ weeks (mean $\pm \mathrm{SD}$ ), birthweight $1.01 \pm 0.20 \mathrm{~kg}$, (mean $\pm \mathrm{SD}$ ), while the seventh was a term infant with necrotising enterocolitis. A combination of intravenous amphotericin B $(0.25 \mathrm{mg} / \mathrm{kg}$ a day increasing to $1.0 \mathrm{mg} / \mathrm{kg}$ a day over 4 days) and flucytocine $100-150 \mathrm{mg} / \mathrm{kg}$ a day was given for 3 weeks followed by 2 weeks of oral flucytocine. Five infants were treated with combined therapy while 2 infants received flucytocine alone. Blood cultures were sterile after 48 hours except in 1 baby in which a $C$. albicans resistant to flucytocine was isolated before the addition of amphotericin $B$. In view of the reported side effects of these drugs all infants had twice weekly blood cultures, full blood count with differential white cell and platelet counts, urea and electrolytes, serum creatinine, and liver function tests. The only abnormalities were a marked increase in alkaline phosphatase and gamma glutamyltransferase in 5 infants. These changes were not appreciably different to those seen in 8 other preterm infants, gestational age $29 \cdot 1 \pm 1.9$ weeks (mean \pm SD), birthweight $0.93 \pm 0.37 \mathrm{~kg}$ (mean \pm SD) on parenteral nutrition without fungal septicaemia.

We would like to suggest that combined amphotericin $B$ and flucytocine therapy is a treatment of choice in neonatal systemic fungal infection and that the above regimen has not led to the side effects reported by others.

\section{References}

1 McDougall P N, Fleming P J, Speller D C E, Daish P, Speidel B D. Neonatal systemic candidiasis: a failure to respond to intravenous miconazole in two neonates. Arch Dis Child 1982; 57: 884-6.

${ }^{2}$ Lilien L D, Ramamurthy R S, Pildes R S. Candida 